\title{
Beitrag zur Schwangerschaft ausserhalb der Gebärmutterhöhle.
}

\author{
Von \\ Dr. Co h n s t e i n, \\ Docent der Gynäkologie an der Universität Heidelberg.
}

Von den in den letzten Jahren in der medicinischen Klinik zu Heidelberg beobachteten extrauterinen Schwangerschaften haben die von Kussmaul und Friedreich beschriebenen Fälle ein allgemeineres Interesse erfahren. Im $\mathrm{K}$ u s s m a u l'schen Falle handelte es sich um Eileiterschwangerschaft mit Degeneration des Chorion (Graviditas tubaria lateris sinistri molaris); der Friedreich'sche Fall höchst wahrscheinlicher Extrauterinschwangerschaft brachte uns als neve Behandlungsmethode die Morphiuminjectionen in das Ei zum raschen Absterben der Frucht. Ich benutze gern die mir von Herm Geh. Rath Friedreich gegebene Erlaubniss, einen weiteren Fall von extrauteriner Gravidität, der sich auf seiner Klinik ereignete und mit vollständigem Erfolge mittelst Morphiuminjectionen von demselben behandelt wurde, eingehender zu veröffentlichen.

Die 25jährige Dienstmagd Susanne Köhler aus Ladenburg war bis Ende 1875 stets gesund. Die im 18. Lebensjahre zum ersten Male eingetretene Menstruation kehrte regelmässig alle vier Wochen wieder, durchschnittlich drei Tage andauernd. Anfangs December 1875 kam Patientin, angeblich in Folge einer Erkältung während der Menses, mit Fieber, Kreuz- und Leibschmerzen, Erbrechen, Durchfall, Harndrang, tympanitisch aufgetriebenem Abdomen ins Krankenhaus. Die äussere und innere Exploration begründete die Diagnose einer acuten Perimetritis, welche in sehr kurzer Zeit durch Opium und Eisblase beseitigt wurde. Am 4. Januar geheilt entlassen und in ihre Heimath zurückgekehrt, erkrankte Patientin von Neuem. Zu 
den wiederum auftretenden Leib- und Kreuzschmerzen gesellte sich eine profuse, acht Wochen lang ohne wesentliche Unterbrechung andauernde Metrorrhagie. Nach ziemlich schneller Erholung kam Patientin Ostern 1876 in Dienst, in welchem sie bis zum 2. März 1877 vollständig gesund geblieben ist. An diesem Tage trat sie mit der Klage uiber Leib- und Kreuzschmerzen, Erbrechen, Obstipation, Harnverhaltung und der Angabe ins Krankenhaus, dass die Regel seit sieben Wochen ausgeblieben und die letzte Menstruation schwächer als gewöhnlich gewesen sei. Die Möglichkeit, schwanger zu sein, giebt Patientin zu; der erste Beischlaf soll Anfangs Januar stattgefunden haben.

Die objective Untersuchung der Iungen, des Herzens, der Leber und Milz der wohlgenährten, nur etwas blass aussehenden Pat. zeigt normale Verhältnisse. Der Leib ist mässig aufgetrieben und auf Druck schmerzhaft. In der Ileo-coecal-Gegend ist der Schall gedämpft tympanitisch; bei der Palpation fühlt man hier in der Tiefe einen mittelgrossen, nicht umschriebenen, elastischen, auf Druck empfindlichen Tumor. Bei der Vaginalexploration ist der Cervix in der Führungslinie leicht zu erreichen, von mittlerer Länge, weicherer Consistenz als im virginalen Zustande; beide Muttermundslippen sind gleich lang, glatt, der äussere Muttermund zu einer Querspalte geöffnet und mit dem Zeigefinger leicht zu erweitern. Im vorderen Scheidengewölbe fühlt man den vergrösserten, anteflectirten, nicht empfindlichen Uteruskörper; den von den Bauchdecken aus fühlbaren Tumor. kann man von der Scheide aus nicht genau rom Uterus abgrenzen.

Dieser Befund in Verbindung mit der Anamnese konnte für Gravidität in den ersten Wochen sprechen, während der Ileocoecaltumor bei der hartnäckigen Obstipation als Fäcalgeschwulst angesehen wurde.

Am folgenden Tage (3. März) trat eine mässige Genitalblutung auf, welche nach zweitägiger Dauer vollständig verschwand und am 11. März erst wieder zum Vorschein kam. Dieselbe wurde am 13. März, dem Tage, wo die Menses zum zweiten Male hätten eintreten sollen, profuser und hielt bis zum 24. März, bald stärker, bald wieder sehwächer werdend, an. Während dieser Zeit waren die Leib- und Kreuzschmerzen heftiger geworden, der Stuhl andauernd retardirt; es bestand Harndrang und Harnverhaltung. Der Coecaltumor nahm trotz wiederholter Lavements und Abführmittel an Umfang und Empfindlichkeit nicht $\mathrm{ab}$, schien vielmehr zuzunehmen. Der anteflectirte Uterus ist jetzt auch antevertirt, sowie auch seitlich nach rechts hin verschoben, die Portio stark nach hinten abgewichen. Ordination: Opiate, Eisblase, Catheterisation.

Am 24. März, also 21 Tage nach Auftreten der ersten Blutung, wurde nach vorausgegangenen heftigeren Leib - und Kreuzschmerzen beim Uriniren eine fleischähnliche Masse aus den Genitalien ausgestossen. Dieselbe bildet einen hohlen, geschlossenen Sack, entspricht genau der Form eines Uterus bicornis, an welchem die beiden Tubarmündungen, der obere Abschnitt des Cervix mit einer dem 
inneren Muttermunde entsprechenden Oeffnung deutlich erkennbar und für eine feine Sonde durchgängig sind. Die Oeffnung des Os internum ist im Durchmesser grösser, als die Eileitermündungen. Die Länge des Sackes beträgt $7 \mathrm{Cm}$., ebensoviel der Breitendurchmesser von einem 'Tubarostium zum anderen, die fleischigen Wände des Sackes sind ungefähr $0,5 \mathrm{Cm}$. dick. Seiner Textur nach stimmt der Sack vollständig ïberein mit einer hyperplastischen Decidua. Seine Aussenfläche ist ranh, uneben, aufgefasert, blutig; die innere, der Höhlung zugekehrte Oberfläche ist weich, ungleichmässig, zeigt einzelne, deutlich durch Furchen gebildete, unregelmässige Felder. Die Höhle enthält einzelne kleine, frische Blutcoagula, keine Spur eines Fötus oder einer stattgehabten Einpflanzung. - Beim Ausspannen des Sackes findet man an beiden, besonders aber an der Aussenfläche zahlreiche feine, kleinere und grössere Oeffnungen, welche das Gewebe meist schräg durchbohren und ein maschig lockeres Gefüge geben. - Auf dem Querschnitte bemerkt man zwei Schichten, die äussere stärkere ist heller gefärbt, die innere und dünnere stark injicirt. Ihre dunkelrothe Farbe ist bedingt durch zahlreiche neugebildete, mit Blutkörperchen vollgestopfte Gefässe und zahllose ausgetretene Blutkörperchen. - Das $Z$ wischendrüsengewebe zeigt in den tieferen Schichten stellenweise deutliche Streifung, Spindelzellen mit getrübtem Inhalte. Zahlreiche grosse, kernhaltige, polygonale, zum Theil mit Fortsätzen versehene, sowie kleinere kugelige Zellen finden sich in den oberen Schichten, während die tieferen mehr kleine runde und spindelförmige, theilweise in dichten Haufen vereinigte Zellen enthalten. Die Drüsenschläuche liegen stellenweise dicht aneinander, sind schräg verzogen, hier und da wesentlich erweitert; 'das Cylinderepithel ist in den unteren Partien deutlich erkennbar, an der Mïndung zum Theil plattenförmig, rund, zum Theil verfettet, auch ganz fehlend wie auf der Oberfläche der Schleimhaut.

Nach Ausstossung des Sackes hörte die Blutung einen Tag ganz auf, am folgenden schon kehrte sie stärker wieder. Auch die Leib-und Kreuzschmerzen, der Harndrang, die Uebelkeit hörten nur vorübergehend auf. In der Regio hypogastrica sinistra wird eine mehr längliche, resistente, ziemlich empfindliche Stelle palpirt. Der Tumor in der rechten Seite, immer noch sehr empfindlich, consistenter als früher, mehr umschrieben, hat etwa Faustgrösse erreicht. Bei der inneren Exploration fühlt man zunächst die in die Führungslinie wieder vorgerückte, verkürzte, stark gelockerte, weiche Portio vaginalis, an welche nach rechts hin sich unmittelbar ein harter Körper anschliesst. Hinter dem Scheidentheile erreicht der Finger eine zweite, weniger consistente, sehr empfindliche Geschwulst, welche bei Druck nach oben den äusserlich fühlbaren Tumor hebt, somit als ein Segment des letzteren aufzufassen ist. Den auf der vorderen Vaginalwand aufliegenden Uteruskörper kann man bei der Empfindlichkeit der Regio pubica nicht genau abgrenzen. Die Scheidenschleimhaut ist gelockert und geschwellt. Die Linea alba zeigt leichte Pigmentirung; aus den Brustwarzen, welche von wohlentwickelten Montyomery'schen 
Drüsen umgeben sind, entleeren sich auf Druck einzelne Tropfen klarer, später colostrumartiger Flüssigkeit.

Am 28. März wurde von G.R.Friedrei ch von der Scheide aus, unter Gegendruck von den Bauchdecken, in das hinter dem Scheidentheile yorragende, dem linksseitigen Bauchtumor entsprechende Gewulstsegment 0,01 Morphium muriaticum mittelst der Pravaz'schen Spritze mit langer Canüle injicirt. Der Kreuz - und besonders Leibschmerz liess ebenso nur momentan nach, wie die Empfindlichkeit des rechts gelegenen Tumors. Schon am nächstfolgenden Tage trat Exacerbation der örtlichen und subjectiven Beschwerden auf. Die Genitalblutung persistirt in mässigem Grade. Der Harn enthält geringe Eiweissmengen, zeitweise auch schleimige Fetzen, in welche zahlreiche Krystalle aus Tripelphosphaten und harnsauren Salzen eingebettet sind. Die sehr hartnäckige Obstipation erforderte grössere Dosen Calomel mit Jalappe. - Durch Palpation lässt sich ein gewisser Zusammenhang zwischen den beiden in den Region. hypogastricae liegenden Tumoren constatiren; während der rechts gelegene von festerer, aber ungleichmässiger Consistenz mit seinem oberen Rande sich eine Handbreit unterhalb les Nabels befindet, zeigt der linksseitige, mehr in die Tiefe ragende Tumor undeutliche Fluctuation. Druckbewegungen von den Bauch. decken aus theilen sich dem in der Scheide befindlichen Finger mit, ebenso kann man bei Druck gegen das Corpus uteri eine mässige Erhebung der Bauchtumoren erzielen. Vom Rectum aus erreicht man das untere Segment eines runden prallen Tumors; der untere $\mathrm{Ab}$ schnitt des Douglas'schen Raumes ist ganz frei, man fühlt keine auffallende Resistenz, nur eine mässige Vergrösserung des einen tiefer herabgetretenen Eierstockes. Auscultation negativ.

Am 12., 14., 18., 21. April wurde von G. R. Friedreich von den Bauchdecken aus mittelst der Pravaz'schen Spritze aus dem fluctuirenden Abschnitte des Abdominaltumors Flüssigkeit aspirirt und Morphium muriaticum 0,01-0,02 injicirt. Bei der ersten Punction wurden nur einige Tropfen, bei den folgenden wurden $2-3$ Spritzen einer sanguinolenten, dünnen Flüssigkeit herausgezogen. Dieselbe enthielt nur wenig Eiweiss, von geformten Bestandtheilen sehr kleine, granulirte, runde Zellen. Plattenepithel und Lanugo waren nicht nachweisbar.

Der Effect dieser Injectionen war ein doppelter. Einmal liess die örtliche Spannung, der Leib- und Kreuzschmerz nach, so dass der Bauch tiefer palpirt werden konnte, andererseits zeigte sich worauf wir besonders hinweisen - schon nach der zweiten MorphiumEinspritzung eine wesentliche Abnahme der rechts und links gelegenen Tumoren. Die Aspiration aus dem kleineren harten Tumor auf der rechten Seite des Abdomen ergab nur Spuren blutig gefärbter Fliissigkeit. Bemerkenswerth ist, dass die Morphiuminjectionen nicht den mindesten Einfluss auf das Sensorium und durchaus keine Reactionserseheinungen zur Folge hatten.

Während sich Patientin vollständig wohl fühlt, die örtlichen Beschwerden ganz aufgehört haben, die Tumoren allmälig abnehmen, 
der anteflectirte Uterus wieder deutlich abzugrenzen ist, exacerbirt am 20. Mai die bis dahin in mässigem Grade ununterbrochen vorhanden gewesene Genitalblutung und hält neun 'Tage lang an. Nach einer Pause von 4-5 Tagen beginnt die Blutung von Neuem und persistirt mit bisweilen recht heftigen Exacerbationen bis zum 22. Juni.

Bei der Palpation fühlt man in der rechten unteren Bauchgegend einen kleinen, unter der Hand leicht weggleitenden Theil; auch bei der Vaginalexploration kann man sich einen kleinen Theil zugängig machen, der bei der Untersuchung vom Rectum aus deutlicher sich als rundlich ovaler Tumor anfïhlt. Am linksseitigen Tumor ist Fluetuation kaum noch nachweisbar, dagegen fublt man hier zeitweise bei tieferem Eindringen der Hand ein Crepitiren, wie beim Andrücken eines diunnen, pergamentartigen Schädels. Die Einführung der Sonde gelang nicht. Beim Herabziehen der Vaginalportion mit dem Häkchen lässt sich der Zusammenhang des linksseitigen Tumors mit dem Uterus constatiren.

Patientin hatte sich so weit erholt, dass sie das Krankenhaus in den nächsten 'Iagen verlassen wollte. Da stellten sich am 23. Juni Abends nach einem anstrengenden Spaziergange heftige Schmerzen im Leibe ein, welche in den nächstfolgenden 'Tagen an Intensität noch zunahmen. Das Abdomen ist aufgetrioben, bei Beriihrung sehr empfindlich, Puls 112, lemperatur 38,8. Stuhl retardirt, Brechneignng, Blutabgang aus dem Uterus. Während gegen den 6. Juli za die meisten Erscheinungen allmälig nachlassen, steigert sich die Empfindlichkeit in der linken Unterbauchgegend zumehmend. Der Unterleib erscheint ungleichmässig ausgedehnt, nach links prominirend. Vom rechtsseitigen Tumor ist nichts nachweisbar, sei es dass er verschwunden oder in den anderen ïbergegangen ist; der linksgelegene hat den Umfang eines kleinen Kindskopfes erreicht, ragt bis zum Nabel empor und lässt in der Tiefe Fluctuation erkennen. Bei der inneren Untersuchung findet man den Uterus anteflectirt, die Scheide von vorn nach hinten etwas plattgedrickt; im vorderen Scheidengewölbe, dem Uterus innig anliegend, das Segment cines elastischen, kugelformigen 'Tumors. Durch ein umfangreicheres, tief' in das Becken ragendes Segment des Tumors ist der hintere Seheidengrund nach abwärts gedrängt. An der, der Banchgeschwulst mitgetheilten, Bewegung nehmen beide Segmente 'Thoil. Dor äussere Muttermund ist geschlossen, die Blutnng sistirt. Keine Gefässgeräusche am Bauche wahrnehmbar.

Der Ernährungszustand der Patientin hat sich wesentlich verschlechtert; sie ist abgemagert, ohne Appetit, sehr anämisch, leidet an abendlichen Fieberexacerbationen und Nachtschweissen.

Am 7. August wurden $85 \mathrm{CCm}$. einer gelbröthlichen, leicht opalisirenden Flüssigkeit durch Einstich mit der Pravaz'schen Spritze in den fluctuirenden Bezirk, ant' der Höhe des Tumors in dex linken Unterbanchgegend, durch G. R. Friedreich entleert. Die Flussigkeit von neutraler bis schwach alcalischer Reaction, spee. Gewicht 1024,50, 
ist sehr eiweissreich $(4-5 \%)$. Lockere gallertige, voluminöse Fibringerinnsel scheiden sich nach einstïndigem Stehen spontan aus. Das Filtrat enthielt geringe Mengen von Globulin; das Serumalbumin fiel zum grössten Theile ohne Ansäuerung beim Kochen aus. Die mikroskopische Untersuchung ergab vereinzelte Rundzellen und rothe Blutkörperchen, keine epithelialen Elemente.

Der Tumor verkleinerte sich nach der Entleerung nicht wesentlich, der flüssige Inhalt ist noch deutlich wahrzunehmen. In Folge andauernder Empfindlicbkeit in der linken Unterbauchgegend täglich subcutane Morphiuminjectionen und warme Umschläge.

Bei der Untersuchung am 28. August findet man eine entschiedene Abnahme des Trumors, dessen Fluctuation nicht mehr nachweisbar ist. Empfindlichkeit ist nur bei starkem Druck noch vorhanden. Bei der Exploratio interna fühlt man den Uterushals weniger stark gegen das Kreuzbein gedrängt, den durch das vordere und hintere Vaginalgewölbe vorragenden Tumor verkleinert. - Allgemeinbefinden besser, Blutung seit neun Wochen sistirt.

Den 7. September. Nur bei längerem Aufbleiben empfindet Patientin, deren Appetit, Verdaung, Schlaf nichts zu wünschen übriglassen, noch Schmerzen im Kreuze und in der linken Unterbauchgegend. Der Leib ist auch bei tiefstem Drucke nicht schmerzhaft. In der linken Unterbauchgegend fühlt man einen apfelgrossen, circumscripten, elastischen, nicht ganz gleichmässigen, mässig beweglichen Tumor. Der Uterus ist anteflectirt, nicht vergrössert, der Scheidentheil leicht zu erreichen. Durch das linke Seitengewölbe der Vagina palpirt man das untere Segment des Tumors, der sich schmerzlos. tiefer nach abwärts verschieben lässt. Fluctuation ist weder von den Bauchdecken, noch von der Scheide aus wahrzunehmen. Der grössere Abschnitt der Geschwulst ragt gegen das Kreuzbein und kann bei tiefem Eingehen in das Rectum umschrieben werden. Auch diese Partie ist ungleichmässig und lässt stellenweise härtere Stellen durchfiihlen. Die Regel ist noch nicht eingetreten; kein Ausfluss.

Den 14. September. Stetige Besserung des Allgemeinbefindens. Der Tumor hat an Umfang nicht abgenommen, erscheint aber wesentlich härter und ungleichmässiger.

Es scheint nunmehr alle Aussicht vorhanden zu sein, dass Patientin bei andauernder Schonung in einigen Wochen das Krankenhaus geheilt verlassen wird. Die Verkleinerung des Tumors

Anmerkung. Am 8. November wurde Patientin, welche inzwischen zwei Mal normal menstruirt und sich vollkommen erholt hatte, von G. R. Friedreich mit der Weisung entlassen, sich nach vier Wochen wieder vorzustellen. Die bimanuelle, vollständig schmerzlose Untersuchung ergab eine Reduction des Tumors auf Wallnussgrösse. 
erfolgt sehr langsam. Während sonst ein Einfluss der extrauterinen Schwangerschaft auf den Uterus noch nach Jahren unverkennbar ist, können wir in unserem Falle eine vollendete Rückbildung annehmen.

Wir haben bei vorliegendem Krankheitsfalle zwei Stadien zu unterscheiden; das erste, welches für uns das meiste Interesse hat, umfasst die Zeit der Entstehung, des Wachsthums und der Abnahme der Bauchgeschwulst; das zweite, die durch vorzeitige mechanische Anstrengung der Reconvalescentin veranlasste circumscripte Peritonitis mit ihren Folgeerscheinungen.

Die im Verlaufe der Krankheit ausgestossene Decidua zeigt, dass wir es bei unserer Patientin mit einom Uterus bicornis unicollis zu thun haben, ähnlich dem von F. C. Naegele dem anatomischen Museum in Heidelberg geschenkten, von Kussmaul in seinem Buche - Vom Mangel der Gebärmutter - Fig. 50, S. 173 abgebildeten Uterus, dessen Längsdurchmesser allerdings kleiner ist.

Die Diagnose der extrauterinen Gravidität lässt sich in den ersten Monaten bekanntlich nie sicher stellen. Wer das Gesammtgebiet der Geburtshülfe und Gynäkologie beherrscht, wird auf dem Wege der Exclusion, der weniger Erfahrene weit eher unter Berücksichtigung der positiven Momente die Wahrscheinlichkeit der Diagnose begrïnden.

Die Anamnese ergab in unserem Falle eine vorausgegangene circumscripte Peritonitis. Ich erinnere daran, dass $\mathrm{Hecker}$ auf die Resultate der partiellen Peritonitiden als auf eine häufige Ursache aller Formen der Graviditas extrauterina aufmerksam gemacht hat.

Bemerkenswerth ist ferner die Wahrscheinlichkeit der Schwängerung, die von unregelmässigen, längere Zeit andauernden Blutflüssen gefolgte Amenorrhoe der sonst regelmässig menstruirten Patientin, die subjectiven Schwangerschaftserscheinungen, welche bei Erstgeschwängerten und gleichzeitiger partieller Peritonitis allerdings keine wesentliche Bedeutung haben. Was das Alter der Patientin betrifft, so sind extrauterine Schwangerschaften im Anfange der zwanziger Jahre zwar selten, aber doch beobachtet worden. Die wehenartigen, mit Stuhl- und Urinverhaltung verbundenen Schmerzen haben jedenfalls nicht die diagnostische Bedeutung, welche ihnen Heim imputirt hat.

Der Krankheitsverla uf bot, wie gewöhnlich, auch in unse- 
rem Falle das Bild einer subacut verlaufenden partiellen Peritonitis.

Die Exploration zeigte Veränderung in den Brüsten Drüsenentwickelung, Secretion - und Pigmentirung der Linea alba. Wir überzeugten uns von der Verkürzung, Lockerung, Schwellung der Vaginalportion, von der Eröffnung des äusseren Muttermundes, der Lockerung und Schwellung der Scheidenschleimhaut, der Zunahme und Vergrösserung der Gebärmutter. Von Bedeutung ist weiter die Lage- und Gestaltveränderung des Uterus, welche sich im Verlaufe der Affection ausbildete und änderte. Eine wichtige, aber nicht immer mit der wünschenswerthen Präcision nachweisbare Erscheinung ist auch die seitliche Abweichung der Gebärmutter. Die bimanuelle Exploration war durch die Schmerzhaftigkeit und Dicke der Bauchdecken, Meteorismus, andauernde Obstipation, Unnachgiebigkeit des Scheidengewölbes und die Bicornität des Uterus erschwert.

Was die Geschwulst betrifft, deren schnelles Wachsthum hervorzuheben ist, so war sie zuerst in der rechten, demnächst in beiden, schliesslich vorwiegend in der linken Unterbauchgegend bemerkt worden. Die Anschauung, dass beide Tumoren der Frucht unmittelbar angebören, vielleicht Kopf und Steiss derselben repräsentiren, liess sich nicht begründen. Einmal war kein directer Uebergang der einen Geschwulst in die andere nachweisbar, andererseits war eine derartige Längsausdehnung der Frucht für die supponirte Schwangerschaftsdauer zu bedeutend, schliesslich waren Consistenz und Wachsthum der beiden Tumoren wesentlich verschieden. Beide Tumoren hingen jedenfalls mit dem Uterus und dadurch auch mit einander zusammen. Wir haben darauf aufmerksam gemacht, dass der rechts gelegene Tumor sich vom Uterus nicht genau abgrenzen liess, dass die Portio vaginalis sich unmittelbar an ihn anschloss. Er erschien von fester, aber ungleichmässiger Consistenz, sein Wachsthum war dem des linksgelegenen Tumors nicht conform; mit der Abnahme des letzteren verkleinerte er sich allerdings auch, aber war nicht mehr palpirbar, als der linksseitige noch deutlich hervortrat. Während der rechtsgelegene Tumor bei der Aspiration nur geringe Spuren blutiger Flüssigkeit ergab, konnten aus dem linksseitigen, von der Scheide und dem Rectum aus gut erreichbaren Tumor, welcher allmälig deutliche Fluctuation zeigte, 2-3 Pravaz'sche Spritzen einer blutigen, dünnen Flüssigkeit 
aspirirt werden. Von Bedeutung erschien uns das, in diesem Tumor zeitweise fühlbare, pergamentartige Crepitiren.

Die rechtsgelegene Geschwulst stellte unserer Ansicht nach das vergrösserte rechte Horn des Uterus dar, während die linksseitige, offenbar hinter und am Uterus gelegene den Fruchtsack repräsentirte. Als in Folge von Morphiuminjection, Aspiration der Flüssigkeit, Tod der Frucht der linksseitige Tumor abnahm, verkleinerte sich - in ähnlicher Weise wie zum Beispiel bei Abdominalschwangerschaft nach Absterben der Frucht der Uterus sich zurückbildet - das rechte Horn. Mit seiner Verkleinerung, mit der Dislocation des Uterus und dem Drucke des Fruchtsackes auf die hintere Uterinfläche steht das Auftreten des kleinen, unter dem Finger leicht weggleitenden Körpers in Zusammenhang, welcher vom Rectum aus als rundlich ovaler Tumor sich anfühlen liess und den wir für das vergrösserte, rechte Ovarium ansprechen möchten.

Was die Punctionsflüssigkeit betrifft, so ist. es überhaupt falsch, wenn man in den ersten Monaten Plattenepithel und Lanugo erwartet. Findet man diese Stoffe doch auch in der ersten Zeit ciner uterinen Gravidität nicht. In einer nicht geringen Zahl von Fällen, welche ich der Literatur entnehme, war die Flüssigkeit, wie in unserem Falle, sanguinolent - in Folge vorzeitiger Ablösung der Placenta.

Ich habe darauf hingewiesen, dass auch bei stärkeren Dosen der injicirten Flüssigkeit keine allgemeinen Morphiumwirkungen aufgetreten sind. Dieses Moment ist bezüglich des Stoffwechsels zwischen Mutter und Kind von Interesse. Wie für gewisse Stoffe ein Uebergang von der Mutter auf den Fötus nicht angenommen werden kann, so scheint auch der Uebertritt von Morphium aus dem fötalen Kreislauf in den der Mutter nicht nachweisbar.

Die Ausstossung der Decidua muss als eins der zuverlässigsten diagnostischen Zeichen der Schwangerschaft ausserhalb des Uterus angesehen werden. $\mathrm{Zu}$ ihrer Auffindung gehören allerdings günstige Umstände, denn die Decidua wird nicht allein, wenn in kleineren Fetzen ausgestossen, sich der Aufmerksamkeit entziehen, sondern auch bei Expulsionen in Continuo übersehen werden, sobald Blutungen vorhanden sind. Bei der Mittheilung aufmerksamerer Patienten, dass sie beim Stuhlgang oder bei der Urinentleerung unter wehenartigen Schmerzen und Blutverlust eine festere Masse verloren, wird der Arzt um so eher einen Abort 
annehmen, als eine längere oder kürzere Amenorrhoe mit Schwangerschaftserscheinungen vorausgegangen, der Muttermund erweitert ist und die Graviditas extrauterina im Verhältnisse zum Abort in den ersten Monaten eine Seltenheit ist. In unserem Falle konnte ein Abort nicht in Frage kommen. Denn da der Sack an seinem unteren Ende geschlossen war und erst aufgeschnitten werden musste, konnten Eihäute und $\mathrm{Ei}$ nicht wohl vorher ausgestossen sein. In den ersten Monaten geht das Ei in der Regel unversehrt ab. Mit der vollständigen Ausscheidung des Eies hätten im Falle eines Abortes die Blutungen nach der Expulsion vollständig aufgehört, während sie im vorliegenden Falle andauerten. Nächstdem war weder eine allgemeine, noch örtliche Ursache nachweisbar, welche zur Annahme eines Abortes berechtigt hätte.

Bei der äusserst seltenen Combination von Graviditas extrauterina mit uterina kann die im Verlaufe der Schwangerschaft ausgestossene Decidua nur Abort sein, wenn es Schwangerschaften derselben Conception sinḑ. ')

Was die Unterschiede zwischen einer bei Extrauterinschwangerschaft und der bei Dysmenorrhoea membranacea ausgestossenen Decidua betrifft, so müssen wir auf den klinischen Verlauf und die Anamnese Rücksicht nehmen. Die Dysmenorrhoea"membranacea tritt im Allgemeinen nur bei Krankheiten und Geschwülsten des Uterus auf, die Expulsion ihrer Decidua erfolgt im Beginne oder im Verlaufe der Menstruation. Der Ausstossung geht keine Amenorrhoe voraus. Die Expulsion der Decidua menstrualis repetirt, im Anschluss an den Menstruationstypus. Eine einmalige Ausstossung der Decidua kommt allerdings vor, gehört aber zu den Ausnahmefällen. Ich habe in der Literatur Fälle von Follin, Lebert, Hegar, Schröder, Huchard, Labadie-Lagrave verzeichnet gefunden; in der Mehrzahl derselben bestanden aber schon vor der Ausstossung die ausgeprägten Sỳmptome einer Metritis. Wenn die Ansicht von Haussmann richtig ist, dass sich die Decidua bei doppeltem Uterus, resp. Extrauterinschwangerschaft durch eine grössere Länge und Dicke vor der Decidua menstrualis auszeichnet, so gehört unsere Decidua der ersten Kategorie an.

Im - zweiten Stadium der Krankheit erregte der fluctuirende Tumor unsere Aufmerksamkeit. Seiner chemischen Beschaffenheit

1) Siehe den Fall von Craghead (Froriep's Tagesb. 1850, Nr. 206). 
nach konnte eine Ovarialeyste ${ }^{1}$, , ein Kystomyom und ein abgekapseltes peritonitisches Exsudat in Frage kommen. Bei allen dreien ist die Flüssigkeit von alkalisch-neutraler Reaction, von hohem specifischen Gewicht und bedeutendem Albumingehalt. Ovarialcystenflüssigkeit schlossen wir aus, da spontane Gerinnselbildung in derselben nur äusserst selten vorkommt und weder Cylinderzellen, noch Körnchenkugeln, Colloidkugeln gefunden wurden. Bei Kystomyomen und peritonitischem Exsudat, bei welchen sich keine organisirten Bestandtheile, höchstens einzelne Blutzellen finden, ist der Gehalt an Fibrin von besonderer Bedeutung. Während bei jenen die spontane Gerinnung $z u$ einer Gallerte, falls sie überhaupt eintritt, in der Regel rapid und massenhaft erfolgt, tritt sie hier langsamer, in unserem Falle nach einer Stunde, ein. Die Kystomyome des Uterus sind bekanntlich immer veränderte Myome. Nun haben wir während der langen Zeit, in welcher die Patientin beobachtet und untersucht worden ist, am Gebärmutterkörper keine Veränderung seiner Form, keine harten Stellen und Höcker wahrnehmen können. Ein Kystomyom kann rasch zunehmen, mir ist aber kein Fall bekannt, in welchem ein Myom so plötzlich entstanden und sich so schnell zu einem Kystomyom verändert hätte.

Auf ein Peritonealtranssudat in Folge entzündlicher Reizung weist die vorausgegangene circumscripte Peritonitis, der Sitz der Geschwulst in der Gegend des Fruchthalters, die plötzliche Entstehung und schnelle Zunahme des Tumors unter den Erscheinungen einer umschriebenen acuten Peritonitis. Es ist nicht unwahrscheinlich, dass die früheren wiederholten Punctionen zur Entleerung des Fruchtsackes an diesen Stellen eine chronische Peritonitis hervorgerufen haben, wie sie Bäumler ( Virchow's Archiv 59, S. 156) beschrieben. Im Allgemeinen sind derartige, durch die Anwesenheit eines Fötus begünstigte Ausgänge einer extrauterinen Schwangerschaft extrem selten. Im Falle von Despine heisst es: „La femme fut atteinte d'hydropisie ascite enkystée peu de temps après le terme de la grossesse et guérit; mais trente ans plus tard une récidive de la même affection eut une issue fatale."

Was die Therapie in den ersten Monaten der extrauterinen

1) Den Ausgang einer Ovarialschwangerschaft in Hydrops ovarii gravidi berichten Fagès (Gaz. méd. 1837, p. 374) und Mayer (Gaz. méd. 1840, S. 246). 
Schwangerschaft betrifft, so empfahl Basedow und insbesondere Kiwisch in Fällen, in denen der abnorme Fruchthalter von der Scheide aus leicht zu erreichen ist, namentlich dort, wo er mehr weniger tief in den Douglas'schen Raum herabgetreten ist, denselben durch den Vaginalgrund mittels eines feinen Troicars zu öffnen und das Fruchtwasser zu entleeren. Seitdem ist die Punction von der Scheide, dem Mastdarme, von den Bauchdecken aus wiederholt ausgeführt worden, und zwar mit ung ünstigem Ausgange von Simpson, Conrad (es wurden etwa $30 \mathrm{Gm}$. frischen Blutes entleert), Martin, Braxton-Hicks, Netzel, Hutchinson, John Scott (die Entleerung der Flüssigkeit gelang nicht), Gallard (mit Wasserinjection), Depaul; mit güns tig em Erfolge zwei Mal von E. Martin (in einem Falle wurden nur wenige Tropfen wässerigen Blutes, im anderen eine geringe Menge theils wässeriger, theils blutiger Flüssigkeit entleert), Greenhalgh, Al. Simpson, Stoltz, Tanner, Koeberlé. Die grössere Anzahl der ungünstig abgelaufenen Fälle weist darauf hin, dass die Punction nur unter bestimmten Cautelen gegen Lufteintritt und Blutung in die Cyste auszuführen ist. Wenn gegen die von Friedreich, Joulin, Cohen zum raschen Absterben des Fötus empfohlenen Morphiuminjectionen in den Fruchtsack eingewendet wird, dass diese Methode keine Vortheile vor der einfachen Punction des Eisackes haben dürfte, so ist der ungünstigen Statistik keine Rechnung getragen. Wälhrend die grössere Punctionsöffnung nicht ungefährlich ist, kann bei minimaler Entleerung von Fruchtwasser die extrauterine Schwangerschaft ungestört weiter verlaufen. Die Morphiuminjectionen haben aber überdies den Vorzug schneller $\mathrm{Ab}$ nahme des Tumors und der localen Beschwerden bei günstigster Prognose für die Mutter.

An den mitgetheilten Fall schliesse ich noch einige allgemeine Bemerkungen zur Lehre von der Schwangerschaft ausserhalb der Gebärmutterhöhle an.

Seit der vorzüglichen und statistisch gewiss brauchbaren Arbeit Hecker's, in welcher zuerst auf die einzelnen Formen der Extrauterinschwangerschaft die gebührende Rücksicht genommen ist, hat sich seit 1859 ein reiches Material durch Publication von Krankengeschichten angesammelt, welches zur Bestätigung und Ergänzung der Hecker'schen Beiträge dienen kann. Wenn auch 
an der Thatsache nicht viel zu rütteln ist, dass die Graviditas abdominalis durch den Verlauf, die Dauer und die Prognose für Mutter und Kind sich wesentlich von den übrigen Formen der extrauterinen Gravidität unterscheidet, so muss man nach den Beobachtungen von Saxtorph, Grenser, Simpson, Fabbri, insbesondere Spiegelberg einräumen, dass auch der Eileiter einer so enormen Ausdehnung fähig ist, um ein ausgetragenes Kind zu beherbergen. Auch bei der Graviditas interstitialis und ovarii kommt nach den Beobachtungen zuverlässiger Autoren Reife des Fötus vor.

Was das Häufigkeitsverhältniss der verschiedenen Formen der extrauterinen Schwangerschaft betrifft, so sind die Angaben von einander abweichend. Kiwisch hält die Schwangerschaft im mittleren Theile der Tuben für die häufigste; ihr zunächst folgt an Frequenz die im äusseren Ende der Tube, dann die primäre Bauchschwangerschaft, demnächst die interstitielle. Nach Hecker's Zusammenstellung kommt die Bauchschwangerschaft absolut häufiger vor, eine auch von Schröder getheilte Annahme. Spiegelberg hält die Tubarschwangerschaft für die häufigste und theilt so die Ansicht Naegele-Grenser's. Nach meiner Zusammenstellung stellt sich das Verhältniss zwischen Abdominal-, Tubar- und Ovarialschwangerschaft im Procentsatze wie $32: 26: 4$, nach de Smet wie $43: 33,5: 11$. Nehmen wir an, dass mehr günstige als ungünstige Fälle publicirt werden, und die grössere Zahl unglücklich verlaufener Fälle sich auf Seiten der Tubarschwangerschaften befindet, so werden die Abdominalschwangerschaften nicht als überwiegend häufiger angesehen werden können.

Für die Ovarialschwangerschaft, welche als solche bekanntlich auch an der Leiche schwer zu erkennen ist, können als. directe Beweise gelten: 1) Das Fehlen des bezüglichen Eierstockes; 2) Verbindung des Sackes mit dem Uterus durch das verlängerte und verdickte Utero-ovarial-Band, Umschliessung des Sackes von dèn beiden Blättern des Ligamentum latum; 3) Lamellöser Bau der Umhüllung und Cylinderepithel der Innenfläche, unmittelbarer Uebergang der Faserzüge der Albuginea in die Wandung des Fruchthalters; 4) In unmittelbarer Nähe der Eiböhle Follikel oder ein Corpus Iuteum, oder kleine Blutcysten. - Die Mehrzahl der als Ovarialschwangerschaft beschriebenen Fälle hat Schrö$d e r$ in der neuesten Auflage seines Lehrbuches citirt. Ich füge 
noch folgende an: Raymond (Annales de Gynécologie, Juli 1874, p. 67), Wiedersberg (Vierteljahrsschrift für praktische Heilkunde 1865, 4), Orth (Beiträge zur Geburtshülfe und Gynäkologie, III, 3. Heft, 1875), als wahrscheinliche Menolti (Annali universali di medicina d'Omodei 1839), Widerste in (Schmidt's Jahrbücher 1853, S. 58), Reymann (Medicinische Zeitung des Vereins für Heilkunde 1859, Nr. 12), Simpson (Edinburgh medical Journal, Septbr. 1863), Neustadl (Prager medicinische Wochenschrift 1864, Nr. 19).

Bezüglich der Menstruation will ich hervorheben, dass verspäteter Eintritt der ersten Menses durchaus nicht selten bei Frauen vorkommt, welche nachträglich an extrauteriner Schwangerschaft erkranken. Was die Menses während der Graviditas extrauterina anlangt, so sind jedenfalls die Fälle am seltensten, in welchen die Menstruation ohne Unterbrechung wiedergekehrt ist, oder von Beginn an durch unregelmässige und häufige Metrorrhagien ersetzt worden ist. Bei Graviditas abdominalis persistirt die Amenorrhoe gewöhnlich 7-9 Monate, bei Graviditas tubaria 4-10 Wochen. Aehnlich sind die Verhältnisse bei Graviditas ovarii, obgleich hier die Angaben in Bezug auf das Ausbleiben der Menses mit dem Alter der Frucht nicht immer im Einklange stehen. Tritt nach einer kürzeren oder längeren Amenorrhoe Blutung ein, so pflegt sie profus, langandauernd, atypisch zu sein. Diese Metrorrhagie hat diagnostisches Interesse. Eine hierauf bezügliche Aeusserung Goupil's lautet: Toutes les malades avaient eu leurs règles supprimées, et la présence seule des métrorrhagies avait pu faire croire à un simple retard de la menstruation, parce qu'on n'avait pas tenu compte des caractères très-différents qui les distinguent des règles, et surtout de leur défaut de périodicité, signe distinctif, sur lequel M. Lachapelle insiste avec raison... Ces pertes ont toujours été regardées, et avec raison, comme un des accidents les plus habituels des grossesses extra-utérines; dans nos observations, les métrorrhagies ont été si fréquentes qu’elles pourraient presque être considérées comme constantes, puisque, dans six observations seulement, elles n'ont pas été notées. Ich habe in ungefähr 80 Proc. der Fälle ${ }^{1}$ ), welche mit darauf bezüg-

1) Martin (Annales de Gynécologie, Juli 1874, p. 71); Raymond, 1. c.; Duguet (Annales de Gynécologie, April 1874, p. 269); Duverney, l. c.; Hennigsen, l. c.; Craghead (Froriep's Notizen 1850, Nr. 206); Leopold (dieses Archiv, X. Bd, S. 248); Walter (Monatsschrift für Geburts- 
lichen Bemerkungen versehen waren, wiederholte, wochenlang andauernde, unregelmässige Blutungen constatiren können, welche zum Theil mit blutig-schleimigem Ausflusse abwechselten, zuweilen von eitriger, stinkender, zäher Flüssigkeit gefolgt waren. Nach Heim's Ansicht sollte der Abgang schleimig und schwärzlich blutiger Flüssigkeit die Tubarschwangerschaft charakterisiren. Eine auch von $\mathrm{Kiwisch}$ hervorgehobene bemerkenswerthe Erscheinung ist die, dass nach dem Absterben der Frucht, sobald keine lebensgefährlichen Zufälle folgen, die früher unterdrückte Menstruation selbst bei stattfindender Retention der Frucht bald wieder regelmässig einzutreten pflegt.

Bezüglich des Alters bestätige ich Hecker's Angaben, dass das dritte Decennium in gleicher Weise bei Tubar - und Abdominalschwangerschaften am stärksten vertreten ist. Die Frequenz im zweiten, dritten und vierten Decennium ist $37: 50: 11,5$.

Das Verhältniss zwischen Erst- und Mehrgebärenden bei den einzelnen Formen der Graviditas extrauterina ist meiner Berechnung nach folgendes:

$\begin{array}{llll}\text { Abdominalschwangerschaft } & 17: 83 \text {, nach Hecker } 14: 86 \\ \text { Tubarschwangerschaft } & 25: 75, \quad, \quad, & 30: 70 \\ \text { Interstitielle } & 38: 62, \quad, \quad, & 19: 80 \\ \text { Ovarielle } & 15: 84 . & & \end{array}$

Das gewöhnliche Verhältniss zwischen Multi- und Primiparen von $3 y_{2}: 1$ ist also nur bei der abdominellen und ovariellen Schwangerschaft zu Gunsten der Mehrgebärenden überschritten.

Die Primiparae sind meist (nahezu $70 \%$ ) alte Erstgebärende

h., 18, S. 188); derselbe (ibidem, S. 201); Conrad (Schweizer Correspondenz-Blatt, IV, 5, 1874); Guichard (Annales de Gynécologie, Mars 1875, p. 224); Leven (Bulletin de la Societé anatomique de Paris 1860, p. 187); Braxton-Hicks (Guy's Hospital Reports, Vol. VIII); Martin (Monatsschrift für Geburtskunde, 21, S. 245); Winckel (Monatsschrift für Geburtskunde, 21, S. 7); Dreessen (Monatsschrift für Geburtskunde, 31, S. 200); Poppel (Monatsschrift für Geburtskunde, 31, S. 208); Pletzer (Monatsschrift für Geburtskunde, 29, S. 242); Fürst (Wiener medicinische Halle 1864, Nr. 9 ff.); Ott (Prager medicinische Wochenschrift 1864, 12); Abarbanell (Monatsschrift für Geburtskunde, 14, S. 188); Schreyer (Monatsschrift für Geburtskunde, 14, S. 283); Dumont-Pall i er (Gazette des hôpitaux 1877, 44); Netzel und Blix (Hygiea 1877, Nr. 3); Roth (Thèse dè Strassbourg 1844, p. 126); Les ouef (l. c. p. 102); Moreau (Des grossesses extrauterinés Paris 1853, p. 110); Gaube (Bulletin de la Société anatomique 1853, S. 120); Aran (Journal des connaissances médicales 1852, p. 548); Queyssac (Encyclopädie, I Ser., t. XXIII); Hennig (dieses Archiv, VI. Bd., S. 138). 
von 30 Jahren und dariiber, welche längere Zeit kinderlos verheirathet waren. Unter den Mehrgebärenden, bei welchen Graviditas extrauterina beobachtet wurde, finden wir meist Frauen, welche 1-3 Mal geboren haben; wesentlich seltener sind Multiparae mit mehr als drei Kindern; am häufigsten vertreten sind diejenigen, welche nur ein Mal recht- oder vorzeitig niedergekommen sind. Die Frauen, welche 1-3 Mal geboren haben, repräsentiren 89,2 Proc., und davon kommen 44,7 Proc. auf die, welche nur ein Mal geboren haben. Eine grössere Fruchtbarkeit scheint also nicht sowohl unter die ätiologischen Momente zu gehören, wie es $\mathrm{Kiw}$ is ch gemeint hat, als vielmehr unter die Präservativmassregeln gegen Eintreten der extrauterinen Schwangerschaft.

Was die Veränderungen im Uterus während der Extrauterinschwangerschaft betrifft, so war die Hyperplasie der Gebärmutter schon älteren Autoren bekannt und ist von D u verney, Santorini eingehender beschrieben worden. Ueber das Vorkommen einer Decidua im Uterus sprach sich zuerst Böhmer aus. (Nihil praeter tenacem et flavescentem mucum, mollemque poroso-villosam et valvulosam quasi turgescentem membranam undique uteri parietes et tubas invertientem, invenimus.) Diese Decidua nennen ältere Schriftsteller, wie Forlanus, Gambini auch Mola, und Uccelli (bei Dezeimeris) beschreibt den Abgang einer fleischigen, hühnereigrossen Mole im dritten Monate einer Extrauterinschwangerschaft.

In der Literatur finden sich sehr zahlreiche, vielfach von einander abweichende Anschauungen über das Auftreten der Decidua, welche ich der Wichtigkeit des Gegenstandes halber, der eine eingehende Besprechung noch nirgends gefunden hat, gern reproducire. Cruveilhier, Bonnet, Bérard behaupten, dass die Decidua ebensowenig constant vorkomme im Verlaufe einer Extrauterinschwangerschaft, wie die Zunahme des Uterus. Nach Baudelocque soll eine Vergrösserung des Uterus nur dann erfolgen, wenn zwischen Gebärmutter und Placenta Beziehungen bestehen. Guillemot stellt das Vorkommen der Docidua mit den, im Verlaufe der Graviditas extrauterina auftretenden Blutungen in Zusammenhang, welche, sobald von Beginn der Schwangerschaft an vorhanden, die Entwickelung der Gebärmutter und Formation der Decidua nicht zu Stande kommen lassen. Beim Ausbleiben der Motrorrhagien fände man einen entwickelten Uterus mit stark vascularisirter Decidua. Nach der Ansicht Lerret's, 
Clarke's, Meckel's, Cazearu's findet eine Formation der Decidua bei allen Formen und in allen Fällen von Graviditas extrauterina statt. "Ceux, qui ont cru ne pas la trouver, quoiqu'ils disent avoir observé un boursoufflement de la muqueuse utérine, se sont trompés et l'ont méconnue; - enfin, dans les cas rares où rien de semblable à une membrane n'a été trouvé dans la cavité utérine, ou bien on a mal observé, ou bien l'examen des pièces a été fait à une époque très avancée de la. grossesse, longtemps après la mort du foetus, lorsque la membrane caduque avait disparu. "

Heim fiihrt unter den Kriterien der Extrauterinschwangerschaft an, dass sich ein röthlicher, schleimiger Ausfluss aus der Scheide entleere, welcher der aufgelösten, abgehenden Tunica decidua zuzuschreiben sei. Nach Vieweg, der die Sondirung des Uterus bei Graviditas extrauterina zuerst empfohlen, geht die Decidua bald in grösseren, bald in kleineren Stücken ab, und zwar bei Graviditas ovaria und tubaria mit der erfolgten Ruptur des Fruchtsackes, oder noch ehe diese eintritt, unter dem Gefühle des Wehendranges. In der Graviditas abdominalis geschieht der Abgang zu der Zeit, wo bei einer gewöhnlichen Schwangerschaft die Fruchttheile ausgetrieben werden. Nach $\mathrm{C}$ a m pbell ist diese Neubildung nicht immer bei allen verschiedenen Formen von Extrauterinschwangerschaft vorhanden, bei Tubarschwangerschaft werde sie übrigens nur selten vermisst. Da jedoch auch in Fällen, in denen sich keine Decidua findet, der Uterus an Volumen zugenommen hat, so muss er wohl denselben Einflüssen, wie sonst, ausgesetzt gewesen sein. Man sei daher zu der Annahme berechtigt, dass die Decidua gebildet worden war und später mit den blutigen Ausscheidungen, oder während der, von Zeit zu Zeit auftretenden, Wehen entfernt worden sei.

Velpeau fasst seine Ansicht dahin zusammen: Les grossesses extra-utérines, la présence de certains polypes et quelques maladies de l'ovaire ou des trompes, déterminent parfois à l'intérieur de l'utérus un boursoufflement, une régétation, un ramollissement, qui ont dû en imposer souvent et faire croire à une caduque véritable. C'est alors qu'on a pu penser que la caduque était organisée, vasculaire, molle, adhérente, percée vis à vis des trompes et du col. - Cependant c'est un état qui n'a aucun rapport avec la caduque normale.

Nach Dezeimeris nimmt der Uterus, wenn auch nicht immer, doch gewöhnlich alle Schwangerschaftszeichen an; er ent- 
wickelt sich so weit, wie etwa in der normalen Schwangerschaft im dritten oder vierten Monate; seine Höhle vergrössert sich, umkleidet sich mit Pseudomembranen, die eine spätere Ausstossung nothwendig machen. - Daraus erklärt sich die Blutentleerung und Ausstossung einer Decidua, welche oft so stark entwickelt ist, dass man sie für die Placenta angesehen hat.

Bei Meissner heisst es: Göppert thut noch eines mit dem Blutflusse aus der Mutterscheide verbundenen Abganges häutiger Massen Erwähnung, der mehrmals beobachtet und durch die Ablösung der, in der Gebärmutterhöhle sich ebenfalls bildenden Membrana decidua erklärt worden ist. Man darf jedoch auch auf diese Erscheinung nicht zu grossen Werth legen, da sie nicht constant ist und sehr leicht zur Verwechselung mit Abort Veranlassung gegeben hat.

Nach Busch wird die hinfällige Haut zuweilen im Verlaufe der Schwangerschaft in eine molenartige Substanz degenerirt. Nach HohI beginnt bisweilen nach längerer Dauer einer Extrauterinschwangerschaft, insbesondere Abdominalschwangerschaft, oder nach Ruptur der Cyste und Austritt der Frucht in die Bauchhöhle, falls die Mutter am Leben bleibt, eine Atrophie des Uterus, so dass er bei der Section leer, ohne Decidua gefunden wird.

Dass der Uterus immer beträchtlich zunimmt und in allen Fällen sich eine Decidua bildet, nehmen $D$ u gès, Kilian, Gardien, Mattei, Hubert, Burns an. Letzterer macht noch besonders darauf aufmerksam, dass die Höhlung der Decidua mit einer Flüssigkeit erfüllt ist, die oft, ja in der Regel, beim Eintritt der Wehen ausgeleert wird, während die Decidua nicht sogleich ausgestossen wird.

In der Epikrise eines Falles von Graviditas tubaria sagt Schwabe:, trotzdem floss aus dem Muttermunde eine amorphe, röthlich gefärbte, klebrige Masse aus, und die Gebärmutterhöhle war damit angefiullt"“. - Er hält diese Masse für das Rudiment der Decidua, die sich, bei ganz anormal entwickelnder Thätigkeit der Geschlechtsorgane zur Ausbildung des Eies, an einem so ungewöhnlichen Orte nicht regelmässig formiren konnte.

Aus der eingehenden Schilderung Kiwisch's hebe ich hervor, dass der Uterus in allen Fällen von. Graviditas extrauterina eine Auflockerung seines Gewebes, eine Vergrösserung seines Umfanges und eine Metamorphose seiner Innenfläche erleidet. Die Decidua, von sehr verschiedener Farbe und Consistenz, ist bald 3-4 ${ }^{\text {Hi }}$ 
dick, bald kaum liniendick. Da sie sich in verschiedenen Zeitabschnitten der Schwangerschaftsdauer abstösst und geboren wird, kommt es vor, dass man sie in einzelnen Fällen mit der übrigen Gebärmuttersubstanz noch innig verbunden antrifft, während sie in anderen Fällen sich ohne besondere Mühe abstreifen lässt, oder sich auch gar nicht mehr vorfindet. Die Ausstossung der Decidua fand in bemerkenswerther Weise bei einzelnen Abdominalschwangerschaften genau am Ende der normalen Dauer einer Schwangerschaft unter heftigem Wehendrange, in anderen Fällen dagegen zu unbestimmter Zeit statt. In einzelnen Fällen fiel letztere mit dem vorzeitig eingetretenen Tode der Frucht zusammen, in anderen dagegen trat die Ausscheidung mitten im Verlaufe der Extrauterinschwangerschaften ein, und endlich fand in den erübrigenden Fällen gar kein bemerkbarer Abgang der Decidua statt. Auch in den Fällen, in welchen der Tod schon in den ersten Monaten plötzlich durch Verblutung erfolgte, fehlte der Deciduaabgang.

Wird die Decidua in einzelnen Fällen vermisst, so hat dies nach Scanzoni darin gewiss seinen Grund, dass man es mit Schwangerschaften aus den späteren Monaten zu thun hatte, wo ja selbst bei der Lagerung des Eies in der Uterushöhle die Decidua an Dicke bedeutend abnimmt. Den Abgang von Deciduafetzen beobachtet man nach Naegele oft schon nach den ersten arei bis vier Wochen. Gelangt das Ei zur vollständigen Reife, dann entleert sich die Decidua auch erst gegen die 40. Schwangerschaftswoche. - In 40 Fällen von Tubarschwangerschaft war nach Hecker der Entwickelung einer Decidua 25 Mal Erwähnung gethan und dabei ein Mal hervorgehoben, dass der Uterus sich wie bei einer Virgo verhalten habe; drei Mal fand sich Vergrösserung des Uterus ohne Bildung der Decidua; eine gänzliche Nichtbetheiligung des Uterus an dem Hypertrophirungsprocesse wurde nur in zwei Fällen beobachtet.

Unter den Ausgängen der Abdominalschwangerschaft citirt Schröder, dass unter wehenartigen Schmerzen nicht selten eine Decidua aus dem Uterus ausgestossen wird. In der neuesten Auflage seines Lehrbuches fehlt das ,nicht selten“, und scheint Schröder demnach die Ausstossung der Decidua als Regel zu betrachten. Auch Depaul will die Exfoliation der Uterinmucosa in allen Fällen beobachtet haben, die er verfolgen konnte. Stets ward die Membran im Ganzen oder in Fragmenten ausgestossen. 
Der Ausstossung der Decidua imputirt Duguet eine üble prognostische Bedeutung, weil in seinem und dem Falle von Pierreson der Expulsion der Tod gefolgt ist. Seiner Ansicht nach veranlassen die Contractionen, welche zur Ausstossung der Decidua führen, auch die Ruptur des Fruchtsackes. Auch nach Spiegelberg sollen die mit Uterinblutung und Abgang von Deciduafetzen verbundenen Schmerzanfälle, besonders wenn sie den Wehencharakter haben, häufig der Vorläufer des tödtlichen Ausganges durch Ruptur des Fruchtsackes sein. Uebrigens hat Spiegelberg in neuester Zeit, im Anschluss an den Fall von ausgetragener Tubarschwangerschaft, den Abgang von Deciduastücken als eins der zuverlässigsten diagnostischen Zeichen der Graviditas extrauterina erklärt.

Was die Veränderungen am und im Uterus während der Extrauterinschwangerschaft betrifft, so können wir auf Grundlage eines recht zahlreichen Materiales folgende Kategorien unterscheiden :

1) Der Uterus ist hyperplastisch, verlängert und breiter; die Decidua haftet seiner Innenfläche entweder fest an, oder befindet sich zum Theil oder vollständig abgestossen in der Gebärmutterhöhle.

Diese Fälle sind die absolut häufigsten.

2) Der Uterus ist deutlich vergrössert, eine Decidua ist nicht vorhanden. Hierher gehören die Fälle von Abarbanell, Ott, Czihak, Loschge, Mattei, Dubois, Bamberger, Bonnet, Gaussail, Matecki, Dreessen, Lesouef. Wir finden den Mangel der Decidua bei allen Formen der Extrauterinschwangerschaft, und können schon um deswillen nicht in allen Fällen an eine vorzeitige Ausstossung derselben denken, weil im Verlaufe der. Gravidität weder Wehen noch Blutungen constant aufgetreten waren, und weil andererseits der Cervicalkanal geschlossen war.

In einer zweiten Reihe von Fällen war die Decidua jedenfalls unbemerkt mit den Blutungen fortgeschwemmt worden, in noch anderen Fällen frühzeitig atrophirt.

3) Das eigentliche Uterusparenchym hat ebensowenig Antheil an der Extrauterinschwangerschaft genommen, wie die Schleimhaut. Das zeigen die Fälle von Saxtorph, Breslau, Gahen, Neustadl, Fleury, Grenser, Smith, King, Murphy, Messer, Pereira, Brackhausen, Davaine. Die Hyperplasie des Uterus kann im 3-4. Monate der Extrauterinschwanger- 
schaft stationär bleiben, oder regressiv abnehmen. Bei Abdominalschwangerschaften, welche das normale Ende erreichen oder übersteigen, bei welchen die Massenzunahme der Gebärmutter angeblich überhaupt keine so bedeutende ist, wie bei Tubar- und Interstitialschwangerschaften, kann aus Rückbildungsprocessen, begünstigt durch den Tod der Frucht, häufige Wehen im Verlaufe der Gravidität, Blutungen, die Intactheit des Uterus und Abwesenheit der Decidua erklärt werden. Fehlt aber bei Autopsien in den ersten zwei Monaten jede Veränderung am Uterus und seiner Innenfläche, so muss man für einzelne Fälle mindestens annehmen, dass die Gebärmutter durch den Schwangerschaftsvorgang gar nicht tangirt worden ist. Auch bei doppelter Gebärmutter findet man, dass die ungeschwängerte Hälfte an den Veränderungen nicht immer Theil nimmt; die Beobachtungen von Cruveilhier, Rokitansky, Busch lehren, dass die beiden Hörner nicht ausnahmlos solidarisch mit einander verbunden sind. - Auch die vielseitig aufgestellte, oben angezogene Ansicht, dass bei Tubarschwangerschaft die Hyperplasie der Gebärmutter bedeutender als bei abdomineller sei, halte ich nicht für ausnahmslos begründet. Gardien will sogar aus der Schwere der Gebärmutter die Art der Extrauterinschwangerschaft bestimmen und meint, dass eine Graviditas abdominalis vorliege, sobald die Schwere des Uterus nicht vermehrt ist. Ich glaube, dass bei Tubarschwangerschaft die Massenzunahme des Uterus sich nur darum leichter documentirt, weil die Section in frühe Schwangerschaftsmonate fällt, während bei Abdominalgravidität die Autopsie meist vorgeschrittene Rückbildung nachweist. Uebrigens kommen bei Abdominalschwangerschaft Längen- und Breitenverhältnisse des Uterus vor, wie sie bei Tubarschwangerschaft nicht beobachtet werden. Hecker fand als Maximum bei Tubarschwangerschaft den Uterus $13,5 \mathrm{Cm}$. lang und $8 \mathrm{Cm}$. breit. In der von Matecki beschriebenen Abdominalschwangerschaft war der Uterus $16 \mathrm{Cm}$. lang und $14 \mathrm{Cm}$. breit, bei Fick $14 \mathrm{Cm}$. lang und $9 \mathrm{Cm}$. breit, bei Simpson $19 \mathrm{Cm}$. lang und $9 \mathrm{Cm}$. breit.

4) Selten sind die Fälle, in welchen der Uterus an der Schwangerschaft keinen Antheil genommen hat, während die Schleimhaut sich in einem gewissen hyperplastischen Zustande befindet. Wir zählen hierher die Fälle von Oldham, Hecker, Hennig.

5) Auch die Fälle, in welchen der Abgang der Decidua im Verlaufe der Schwangerschaft bemerkt und berücksichtigt worden 
ist, sind ziemlich selten. Ich habe in einem casuistischen Materiale von 600 Krankheitsfällen folgende Bemerkungen auffinden können :

1) Vieweg (Nene Zeitschrift für Geburtskunde, VIII, 328): Graviditas abdominalis. Im 7.-8. Monate Abgang einiger Stiicke. - Ausgang für die Mutter günstig.

2) Derselbe (ibidem): Graviditas ovaria. Im 3.-4. Monate unter profusen Blutungen. - Ungünstig.

3) Adams (Medical Times, 24. Novbr. 1860): Graviditas abdominalis. Am normalen Ende stückweise unter Blutungen. Günstig.

4) Dugu et (Annales de Gynécologie 1874, p. 269): Graviditas tubaria. Im 3.-4. Monate wird ein fast vollständiger Ausguss der vergrösserten Uterinhöhle unter Wehen und Blutungen ausgestossen. Es fehlt nur eine kleine Partie in der Gegend einer Tubarinsertion, welche im Uterus zurückgeblieben ist. - Vier Tage nach der Ausstossung Tod.

5) Pier reson (Bulletin de la société anatomique 1861, p. 25): Graviditas abdominalis. Im dritten Monate Ausstossung einer vollständigen Decidua (ohne Blutung), der nur ein im Uterus zurückgebliebenes Stück fehlt. - Zwei Tage darauf Tod.

6) Duverney (Oeurres anatomiques, t. II., p. 355): Graviditas tubaria. Im $2^{1 / 2}$ Monate unter Wehen und Blutungen. - Tod.

7) Hennigsen (dieses Archiv, I. Band, S. 334): Graviditas. abdominalis. Am normalen Ende unter Wehen und Blutungen. Decidua von der Form und Grösse eines Gänseeies. - Genesung; drei Jahre später Tod durch Verjauchung des Fötus.

8) Rosshirt (Neue Zeitschrift für Geburtskunde IX, S. 400): Graviditas tubo-uterina. Am Ende der Schwangerschaft unter Wehen kleine häutige Stücke. - Ausgang ungünstig.

9) Frohberg (Neue Zeitschrift für Geburtskunde I, S. 134): Graviditas abdominalis. Am Ende der Schwangerschaft werden 4-5 Stücke, jedes von ungefähr einer Unze Gewicht, einer thymusähnlichen Masse entleert. - Gastrotomie. Tod.

10) Spiegelberg (dieses Archiv, I. Band, S. 408): Graviditas tubaria. Am Ende der Schwangerschaft werden Deciduafetzen mit dem in den Uterus eingefïhrten Katheter entleert. - Tod.

11) Hecker (Monatsschrift für Geburtskunde, 13. Bd., S. 119): Graviditas abdominalis. Im siebenten Monate der Schwanger- 
schaft werden schmierige und aashaft stinkende Deciduafetzen mit dem Finger aus dem Muttermunde entleert. - Tod.

12) Leopold (dieses Archiv, X. Band, S. 248): 'Graviditas tubaria. Im vierten Monate hellröthlicher, übelriechender Ausfluss, der hier und da kleine Schleimhautfetzen enthielt. - Tod im vierten Monate.

13) Schleifer (Medicinische Jahrbücher Oestreichs. Neue Folge, XVI, S. 503): Graviditas abdominalis. Im vierten Monate unter Wehen und Blut Abgang einer häutigen Mola. - Drei Wochen darauf Ruptur per rectum. Genesung.

14) Nouveau Journal de médecine, chirurgie et pharmacie, Vol. XV, p. 51: Graviditas abdominalis. Am Ende der'Schwangerschaft Abgang einer molenähnlichen Masse. Laparotomie. Mutter todt, Kind lebt.

15) Turnbull (Memoir of the medical society of London, Tom III, p. 231): Graviditas abdominalis. Abgang der Decidua am Ende der Schwangerschaft.

16) Campbell (Abhandlungen über Extrauterinschwangerschaft, Karlsruhe 1841, S. 68): Graviditas abdominalis. Im vierten Monate Abgang einer molenähnlichen Masse. - Tod im neunten Monate.

17) Bonnie (bei Campbell, S. 85): Graviditas abdominalis. Im dritten Monate faustgrosser Körper. - Perforation per rectum. - Tod.

18) Uccelli (bei Dezeimeris): Graviditas abdominalis. Im dritten Monate Abgang einer fleischigen Mole. - Tod im vierten Monate.

19) Jobert de Lamballe (Gazette des hôpitaux 1845, Nr. 78): Graviditas abdominalis. Abgang der Decidua am Ende der Schwangerschaft. - Günstig.

20) Walter (Monatsschrift für Geburtskunde, 18, S. 192): Graviditas abdominalis. Vierzehn Tage nach dem normalen Ende wird viel Blut mit viel faserigen und membranösen Stäcken entleert. - Ausgang günstig.

21) Derselbe (ibidem, p. 202): Graviditas. (?) Mit schleimigeitrigen Blutungen werden membranöse Massen im vierten Monate entleert. - Ausgang günstig.

22) Conrad (Schweizer Correspondenzblatt IV, 5, 1874): Graviditas abdominalis. Im dritten Monate gingen unter starken 
Schmerzen häutige Fetzen mit Blut ab; darauf stinkender Ausfluss. - Ausgang günstig.

23) Derselbe (ibidem): Graviditas abdominalis. Einen Monat nach dem normalen Ende ging unter wehenartigen Schmerzen und ziemlich bedeutender Blutung ein rundlicher, fleischartiger Körper von der Grösse eines Hühnereies $a b$, einige Tage später ein kleineres Stück von ähnlichem Aussehen. - Ausgang günstig.

24) Guichard (Annales de Gynécologie 1875, p. 224): Graviditas abdominalis. Im neunten Monate unter Blutungen und Wehen. - Ausgang ungünstig.

25) Féré ol (L'Union médicale 1875, 4. Decbr.): Graviditas. (?) Im zweiten Monate wird die trianguläre, dicke, villöse. Decidua ohne vorausgehende, noch nachfolgende Blutung ausgestossen. Genesung.

26) Goldberger (Oestreichische medicinische Jahrbücher 1841, S. 183): Graviditas abdominalis. Am normalen Ende. - Tod.

27) Levy (Bibliothek for Lager 1860, Octbr.): Graviditas abdominalis. Im 7.-8. Monate Abgang einer fauligen, fleischähnlichen Masse unter übelriechendem, ein Jahr andauerndem Ausflusse. - Tod.

28) Martin (Monatsschrift für Geburtskunde, 21, S. 245): Graviditas abdominalis oder ovaria. Im 4.-5. Monate werden mit bedeutenden Blutungen häutige Massen ausgéstossen. - Genesung.

29) Martin (Monatsschrift für Geburtskunde, 31, S. 248): Graviditas tubaria. Im dritten Monate unter Blutung ein Stïck Decidua abgegangen. - Genesung.

30) L. Mayer (Monatsschrift für Geburtskunde, 21, S. 25): Graviditas abdominalis. Im dritten Monate unter mässiger Blutung. - Ungünstig.

31) Greenhalgh (Lancet, 23. u. 30. März 1867): Graviditas tubaria? Im dritten Monate, 20 Tage nach der Punction des Fruchtsackes von der Scheide aus, Abgang eitriger Massen und Deciduafetzen unter Wehen. - Heilung.

32) Pletzer (Monatsschrift für Geburtskunde, 29, S. 242): Graviditas abdominalis. Im achten Monate Entleerung einer vollständig ausgebíldeten Decidua unter Wehen und sehr geringem Blutverluste. - Tod.

33) Weber-Ebenhof (Wiener medicinische Presse 1867, 
Nr. 50/51): Graviditas abdominalis. Am Ende der Schwangerschaft fühlt der explorirende Finger im Muttermunde Deciduafetzen. - Tod.

34) Haselberg (Beiträge zur Geburtshülfe 1874, 3. Band, 2. Heft): Graviditas ovarii. Im zweiten Monate wurde unter mässiger Blutung eine fingerdicke, circa $2 "$ lange Decidua ausgestossen. - Tod am Ende des zweiten Monates.

35) Wegscheider (Verhandlungen der Berliner geburtshülflichen Gesellschaft, IV, S. 3): Graviditas tubaria. Mehrere Tage nach Berstung des Fruchtsackes Abgang der Decidua.

36) Schröder (Dieses Archiv, V. Bd., S. 354): Graviditas tubaria. Abgang einer deutlichen Haut am 10. Tage nach Anreissung des Fruchtsackes. - Tod 17 Tage nach der Expulsion. Haematocele retro- und anteuterina.

37) Van Peene (Annales de la Société de Gand, t. 43, p. 34): Graviditas abdominalis. Am Ende der Schwangerschaft wird unter heftigen Wehen ein vollständiger Ausguss des Uterus ausgestossen. - Tod.

38) Revillout (Gazette des hôpitaux 1867, Nr. 146): Graviditas tubaria. Aus der Scheide ging eine ganze Decidua ab, der nur eine Stelle fehlte, die etwa dem einen Horne des Uterus entsprach. Der Uterus enthielt in seinem linken Horne den Rest der Decidua. - Tod.

39) Gusserow (Dieses Archiv, 12. Bd., S. 76): Graviditas abdominalis. Im achten Monate - 18 Tage nach dem Tode der Frucht - Abgang der Decidua unter starken Blutungen. - Laparotomie. - Günstiger Ausgang.

Der Behauptung, dass die Ausstossung der Decidua ein gewöhnliches, oder nur sehr häufiges Vorkommniss im Verlaufe der Extrauterinschwangerschaft sei, muss ich entschieden entgegentreten, obgleich ich einräume, dass ein Procentsatz von 6,5 (d. h. auf $600-39$ ) der Wirklichkeit nicht entspricht, weil besonders dünne, leicht zerreissliche, in Stücken abgehende Deciduen häufig übersehen werden.

Die Ausstossung der Decidua findet sich bei allen Formen der Extrauterinschwangerschaft, am seltensten - entsprechend der Seltenheit der Fälle überhaupt - bei interstitieller Schwangerschaft; nahezu gleich häufig bei Tubar- und Bauchschwangerschaft. 
Bei Abdominalschwangersehaft erfolgt die Ausstossung am häufigsten gegen Ende der normalen Schwangerschaftsdauer, in Ausnahmefällen nach dem normalen Ende. Aber auch zwischen dem 3.-4. und dem 7.-8. Graviditätsmonate ist die Ausstossung durchaus nicht selten. Die Ausstossung coincidirt mit den Wehen, welche $\mathrm{zu}$ jeder Zeit, am häufigsten in den angegebenen Monaten auftreten. Bei Tubarschwangerschaft erfolgt die Ausstossung gewöhnlich nach erfolgter Ruptur oder Anreissung des Fruchtsackes.

Aus der Beschaffenheit einer in den ersten Monaten abgehenden Decidua lässt sich die Form der Extrauterinschwangerschaft nicht diagnosticiren, da sich sowohl bei Abdominal - wie Tubarschwangerschaften die Decidua in einzelnen Fetzen, als geschlossen und zusammenhängend entleert.

In der Regel erfolgt die Ausstossung unter Wehen und mit Blutungen, bisweilen - insbesondere bei stückweiser Expulsion mit eitrigem, übelriechenden Ausflusse. Die Ausstossung wird begünstigt durch Punction des Fruchtsackes, Einführen des Fingers oder der Sonde in den Uterinkanal. Mit der Ausstossung der Decidua treffen nicht selten zusammen Zeichen von Absterben der Frucht, Abnahme der Bauchgeschwulst, Abschwellen der Brüste, bisweilen auch Eintritt der Milchsecretion, momentane Besserung des Allgemeinbefindens. Das primum movens scheint der Tod der Frucht zu sein und die Ausstossung der Decidua bedingt durch die, dem Absterben der Frucht folgenden, Contractionen des Fruchtsackes und des Uterus. Die Decidua ist ein Zeichen des regressiven Vorganges.

Dass die Expulsion eine üble prognostische Bedeutung haben kann, lehren die - allerdings nicht sehr zahlreichen - Fälle, in welchen unmittelbar oder einige Tage nach der Ausstossung der Decidua der Tod eingetreten ist. Immerhin muss man, wenn der Tod nicht eintritt, an die Möglichkeit eines intraperitonealen Blutergusses denken. Ich stimme darin vollständig mit J. Veit überein, der noch neuerdings den Zusammenhang der Haematocele mit der Tubenschwangerschaft hervorgehoben hat.

Die Expulsion der Decidua halte ich für das zuverlässigste diagnostische Symptom der extrauterinen Schwangerschaft. Die Ausstossung erfolgt aber nur relativ selten, während das Zurückbleiben der adhärenten oder gelösten Decidua im Cavo uteri als Regel angesehen werden muss. In Berücksichtigung dieses Umstandes glaube ich, dass wir in Fällen, in welchen durch Ein- 
führung der Sonde in den Uterus der Nachweis der Leere der Uterinhöhle gebracht werden soll, mit grösserem Nutzen die Cürette anwenden werden. Denn die Einführung der Sonde in den Uterus ist nicht entscheidend für die Leere der Gebärmutter, während die Entfernung von Deciduafetzen aus dem Uterus positiv für extrauterine Schwangerschaft spricht.

6) Der Schleimpfropf im Cervicalkanale kommt ebensowenig constant vor, wie die Eröffnung des äusseren Muttermundes unter dem Einflusse der Wehen bei extrauteriner Gravidität.

Bezüglich des operativen Eingriffes bei ausgetragener extrauteriner Schwangerschaft kann ich mich um so kürzer fassen, als Spiegelberg in seinem Lehrbuche sehr präcise Vorschriften giebt und Gusserow die Frage in jüngster Zeit unter Berücksichtigung der neuesten Literatur behandelt hat. Der Laparotomie stellt er allerdings nur das exspectative Verfahren gegenüber, während die Elytrotomie übergangen ist. Meiner Ansicht nach hat der Schnitt durch das Scheidengewölbe auf den Fruchtsack mit Entfernung des Fötus schon deshalb eine Zukunft, weil die Jauche, welche beim Zurücklassen von Placenta und Fruchtsack ja nicht zu umgehen ist, wesentlich leichter und vollkommener abfliessen kann. In der Jauchung liegt aber gerade die Hauptgefahr bei der Laparotomie. Auch bei spontanem Durchbruche der Frucht durch die Scheide ist die Prognose für die Mutter wesentlich günstiger, als nach Ausstossung der Frucht durch die vordere Bauchwand. Im letzteren Falle stellt sich nach $\mathrm{Puech}$ der Procentsatz der Genesungen auf 31, im ersteren auf 78. Ein bestimmtes Urtheil über den Vorzug der Elytrotomie gegenüber der Laparotomie kann nur eine reichhaltige Statistik geben; die von $\mathrm{Cau}$ wenberghe - auf 11 Gastrotomien 6 Heilungen, auf 7 VaginoSectionen 4 Todesfälle - und Hecker - auf 16 Gastrotomien 11 Heilungen, auf 5 Vagino-Sectionen 2 Todesfälle - gegebenen Zahlen sind zu gering. Finden wir doch bei einer Zusammenstellung von vierzehn Fällen (Normand, Caignon, Delisle, Kiner, Harder, John King, Burns-Sabatier, Voillemier, Dubois, Simpson, Rupin, Keith, Thomas, Philippart), dass zehn Mütter gerettet und vier gestorben sind. Von zwölf Kindern - ich rechne zwei Fälle $a b$, in welchen die Elytrotomie vor Lebensfähigkeit des Fötus ausgeführt worden ist 
- wurden nur zwei gerettet; ein drittes, lebend geboren, starb eine halbe Stunde post partum. Die sogenannte primäre Elytrotomie wurde zehn Mal, die secundäre vier Mal ausgefübrt. Für die Prognose der Mutter war die erstere günstiger. Im Vergleiche mit den bekannten Zusammenstellungen von Keller und Parry ist die Elytrotomie für die Mutter günstiger als die Laparotomie, im Interesse der Kinder aber letztere vorzuziehen. Indicirt wäre die Elytrotomie bei nicht ausgetragener, bei abgestorbener und tief in die Beckenhöhle herabgetretener Frucht; contraindicirt, wenn die Rettung des Kindes beabsichtigt ist und ferner unter allen Umständen, wenn der Beckeneingang noch nicht passirt ist, weil die für die Extraction nothwendigen Wehen fehlen. - Aeltere Fälle von ausgeführtem Vaginalschnitte citirt $\mathrm{Czi}$ hak (Beiträge von Scanzoni 1860, S. 167).

15. September 1877. 